\title{
Analysis of a Class-B Microwave-Photonic Link Using Optical Frequency Modulation
}

\author{
Peter F. Driessen, Senior Member, IEEE, Thomas E. Darcie, Fellow, IEEE, Member, OSA, and
} Jinye Zhang, Member, IEEE, Member, OSA

\begin{abstract}
A class-B microwave-photonic link using optical frequency modulation (FM) and fiber-Bragg gratings (FBGs) is analyzed and results verified with measurements. Input voltage is converted linearly to FM, and this FM is converted by each FBG to intensity modulation. For small FM index $\beta$, the signal increases as $\beta^{3}$, becoming linear in $\beta$ with a frequency offset or carrier leakage, and third-order distortion resulting from the detection process is shown to be worse than that obtained using a Mach-Zehnder intensity modulator at the same modulation index. For large $\beta(>10)$, third-order distortion approaches zero and ideal class-B operation is predicted, and the only shot noise and relative intensity noise (RIN) added is that which arises from the detected signal power.
\end{abstract}

Index Terms-Class B, microwave-photonic link (MPL), optical frequency modulation (FM), optical link, radio over fiber, spur-free dynamic range.

\section{INTRODUCTION}

$\mathbf{M}$ ICROWAVE-PHOTONIC links (MPLs) play an important role in many applications including video distribution networks, radio-over-fiber, and radar systems. In each application, noise and distortion limit the spur-free dynamic range (SFDR) of signals that can be transmitted [1]. Increasing the dynamic range requires improving the linearity of the RF-tooptical converter, which increases the high-power limit to the SFDR, and reducing noise (intensity, shot, and receiver noise), which reduces the minimum detectable signal.

For traditional links using optical intensity modulation (IM) and direct detection, the high-power limit to the SFDR is limited by the linearity of the input voltage-output intensity transfer function of the modulated optical source. For low-frequency $(<1 \mathrm{GHz})$ applications (e.g., hybrid fiber-coax cable television networks), direct modulation benefits from the intrinsic linearity of a laser current-output intensity response [2]. At higher frequencies, where carrier dynamics from direct modulation create undesirable resonance distortion, external modulation is used. Most existing high-performance links use Mach-Zehnder (MZ) modulators [3]. Although the sinusoidal MZ voltage-transmission transfer function introduces undesirable third-order distortion, the predictability of the response is well suited for electronic predistortion [4]. Electroabsorption modulators (EAMs) offer small size and low power [5], and may provide high linearity under limited operating conditions.

Manuscript received January 30, 2008; revised June 5, 2008. Current version published October 10, 2008. This work was supported by the NSERC.

The authors are with the Department of Electrical and Computer Engineering, University of Victoria, STN CSC, Victoria, BC V8W 3P6, Canada (e-mail: peter@ece.uvic.ca; tdarcie@uvic.ca; jinzhang@uvic.ca).

Digital Object Identifier 10.1109/JLT.2008.927217
Even for a perfectly linear IM response, the high-end of the SFDR is limited by clipping, when the input voltage exceeds the maximum available optical power. This has been studied for cable signals [6] and more recently for MPLs [7], revealing that existing links fall far short of fundamental limits for linearity. Hence, effort continues to define alternative methods for providing high end-to-end system linearity.

Popular recent approaches include the use of FM or phase modulation (PM) with various forms of frequency or phase detectors. An interferometer can be used to detect PM, which may have several practical advantages [8], but otherwise preserves the sinusoidal transfer function of a MZ modulator. PM/FM can be detected using specially-fabricated FBGs [9], where the performance becomes defined by the fabrication of the gratings. Finally, PM can be detected using an optical phase-locked loop (OPPL) [10]. Under conditions of high loop gain, high linearity is achievable as the OPPL tracks the PM on the received optical signal. Loop stability is a challenge for high gain. So collectively, these approaches struggle to extend the SFDR at the high input signal limit by reducing distortion.

At the low input signal limit, noise reduction can also lead to large improvements in SFDR. Shot noise and relative intensity noise (RIN) powers scale linearly and quadratically in received optical power, respectively. Since for typical linear operating conditions much of the received optical signal power resides in the optical carrier, carrier suppression becomes an important objective. Examples of approaches explored to suppress the carrier include low-biasing an MZ [11], which reduces the response of the signal, and filtering the optical carrier [12], which adds distortion. Also, the use of PM/FM and FBGs can provide good linearity at low bias [9].

To maximize SFDR, it is essential to address both the high and low input power limits to the SFDR. This requires joint carrier suppression and linear modulation. An example of this was demonstrated [13], and extended into class $\mathrm{AB}$ [14]. This involves the use of complementary low-biased links to provide an effectively linear transfer function with zero average dc effective photocurrent. Ideally for class B, each link would half-wave rectify the input signal and the complete signal would be reconstructed at the output of a balanced photodetector. An ideal class B link has been analyzed [15], in which the only shot noise and RIN added is that which arises from the detected signal power, which can be well below receiver noise.

Unfortunately, while class B offers potentially high performance, implementation is challenged by the absence of an ideal linear threshold converter (ILTD). The ILTD must provide an output optical power of zero for input voltage $>0$ (or $<0$, for the 


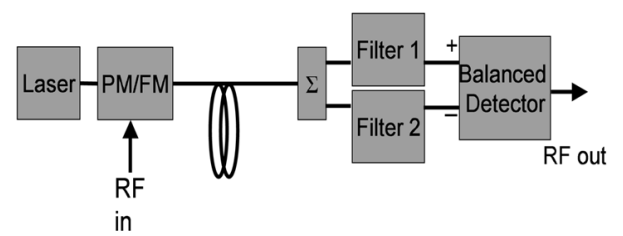

Fig. 1. Class B optical link with PM/FM modulation, two FBGs, and balanced (differential) detection.

complementary ILTD), and linear response for voltages $<0$ (or $>0$ ) up to the maximum optical power available from the source. While direct modulation of a laser diode appears to match this requirement, operating a laser diode biased at threshold introduces many problems. These include distortion from gain dynamics and high RIN.

In an attempt to overcome this limitation, class B has been explored using FBGs and PM/FM [16]. In principle, input voltage is converted linearly to optical frequency at the transmitter, then a FBG with a reflectivity that is linear with optical frequency is used to provide linear modulated intensity. The resultant filtered optical electric field is square-law detected in a photodetector to produce electrical current. This differs from the standard FM frequency discriminator used in radio systems, in which the input frequency is discriminated to produce voltage (electric field) without the square-law detection. It also differs from normal (class A) optical FM-to-IM conversion using an optical filter (in which the filtered signal is square-law detected), in that the nonlinear threshold in the class-B filter design adds a dramatic complication of both analysis and behavior. This leads to complex interactions between FM conditions, FBG characteristics, and FBG bias conditions.

In this paper, we present a complete theoretical description of this detection/discrimination process and related forms of detection of angle-modulated optical signals. Predictions are confirmed through experimental measurement for a variety of modulation and bias conditions. The paper is organized as follows. In Section II, the requirements for class B in the context of an optical system are described. Expressions are derived for the output of the two FBGs with an FM signal input. In Section III, the experimental setup is described, followed by numerical and experimental results in Section IV, discussion in Section V, and conclusions in Section VI.

\section{ANALYSIS}

Fig. 1 shows the class B optical link with two FBGs and a balanced detector [16]. We now describe the FM signal and filter characteristics, yielding a general expression for the output of each FBG.

\section{A. Formulation}

An angle modulated signal with optical carrier $w_{c}$, microwave signal $w_{m}$, and modulation index $\beta$

$$
\begin{aligned}
s(t) & =\cos \left(w_{c} t+\beta \sin w_{m} t\right) \\
& =\sum_{n=-\infty}^{\infty} J_{n}(\beta) \cos \left(w_{c}+n w_{m}\right) t .
\end{aligned}
$$
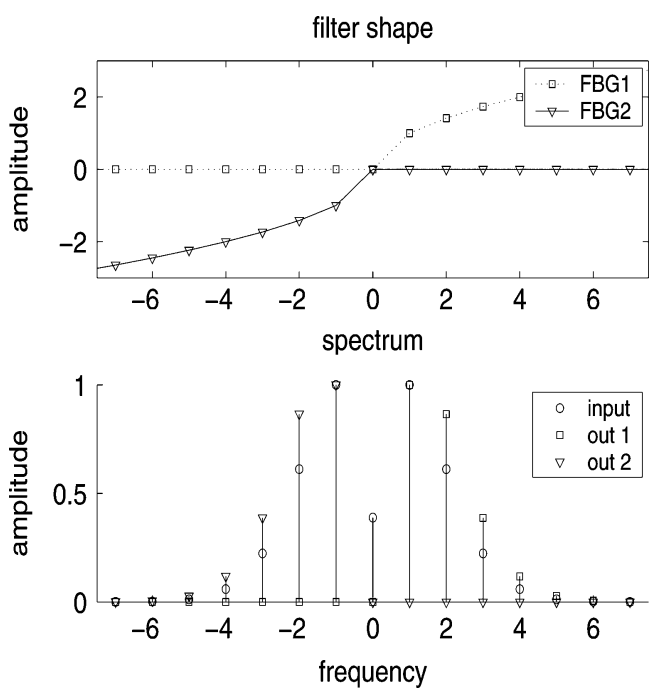

Fig. 2. (a) FBG amplitude response. The FBG power response is linear in the operating range. (b) FM magnitude spectrum at FBG input and output for $\beta=2$. The frequency in $\mathrm{GHz}$ is relative to the optical carrier.

With only a single microwave carrier at a constant $w_{m}, s(t)$ may be viewed as both an FM and a PM signal, with $\beta$ interpreted as the phase deviation as well as the frequency deviation divided by $w_{m} . s(t)$ is converted by the two FBGs FBG1, FBG2 to intensity modulation (IM).

FBG1 may in general be represented as a filter as shown in Fig. 2(a), such that at the discrete frequencies $w_{c}+n w_{m}$ present in the spectrum of $s(t)$, the filter output magnitude is $h_{n}=$ $\left|H\left(w_{c}+n w_{m}\right)\right|$ and with constant group delay $\tau$.

The output of FBG1 is

$$
\begin{aligned}
r_{1}(t) & =a_{1}(t) \cos \left[w_{c} t+\phi_{1}(t)\right] \\
& =\sum_{n=0}^{\infty} h_{n} J_{n}(\beta) \cos \left[\left(w_{c}+n w_{m}\right)(t-\tau)\right]
\end{aligned}
$$

with detected photocurrent (optical power)

$$
\begin{aligned}
a_{1}^{2}(t)= & {\left[\sum_{n=0}^{\infty} h_{n} J_{n}(\beta) \cos \left(n w_{m} t-\left(w_{c}+n w_{m}\right) \tau\right)\right]^{2} } \\
& +\left[\sum_{n=0}^{\infty} h_{n} J_{n}(\beta) \sin \left(n w_{m} t-\left(w_{c}+n w_{m}\right) \tau\right)\right]^{2}
\end{aligned}
$$

and phase

$$
\phi_{1}(t)=\arctan \left(\frac{\sum_{n=0}^{\infty} h_{n} J_{n}(\beta) \sin \left(n w_{m} t-\left(w_{c}+n w_{m}\right) \tau\right)}{\sum_{n=0}^{\infty} h_{n} J_{n}(\beta) \cos \left(n w_{m} t-\left(w_{c}+n w_{m}\right) \tau\right)}\right) .
$$

The output of FBG2 is described by the same expressions with the summation from $-\infty$ to 0 and $h_{-n}=h_{n}$. To find the spectrum of $a_{1}^{2}(t)$, we write $j_{n}=h_{n} J_{n}(\beta)$ for convenience, expand both summations, compute the square of each, add them and simplify using $\cos (u) \cos (v)+\sin (u) \sin (v)=\cos (u-v)$.

The result is

$$
a_{1}^{2}(t)=\sum_{i=-\infty}^{\infty} j_{i}^{2}+2 \sum_{k=1}^{\infty} \sum_{i=-\infty}^{\infty} j_{i} j_{i+k} \cos \left(k w_{m}(t-\tau)\right)
$$


As a check, we consider two special cases for the filter $h_{n}$. For an all-pass filter $h_{n}=1, j_{n}=J_{n}(\beta)$, there is no discrimination or demodulation, so we expect the envelope $a_{1}^{2}(t)$ to be 1. Since $\sum_{-\infty}^{\infty} J_{n}(z)=1$, the dc term is 1 . Since $J_{-n}(x)=$ $(-1)^{n} J_{n}(x)$, all terms at odd multiples of frequencies $n w_{m}$ are zero. All terms at even multiples are also zero, using the identity [17]

$$
0=\sum_{k=0}^{2 n}(-)^{k} J_{k}(z) J_{2 n-k}(z)+2 \sum_{k=1}^{\infty} J_{k}(z) J_{2 n+k}(z) .
$$

As a second check, if $h_{n}=K \sqrt{n+n_{1}}$ so that $j_{n}=K \sqrt{n+n_{1}} J_{n}(\beta)$, where $n_{1} \gg 1$ is such that $J_{n}(\beta) \simeq 0$ for $n>n_{1}$, and $K>0$ is some constant, then we expect class A demodulation. We have numerically verified the envelope $a_{1}^{2}(t)$ to be dc plus $\cos \left[w_{m}(t-\tau)\right]$ with negligible higher harmonics $(<-100 \mathrm{~dB})$, as expected.

For the FBGs considered here, the amplitude response in Fig. 2(a)

$$
\begin{aligned}
H_{1}(w) & =0, w<w_{c} \\
& =g \sqrt{\left(w-w_{c}\right)} e^{j w \tau}, w_{c}<w<w_{c}+n_{0} w_{m} \\
& =e^{j w \tau}, w>w_{c}+n_{0} w_{m}
\end{aligned}
$$

where the constant $g=1 / \sqrt{n_{0} w_{m}}$ is chosen so that the filter gain is 1 at $w=w_{c} \pm n_{0} w_{m}$ for some $n_{0}$ where there are no significant sidebands in $s(t)$. From Carson's rule $n_{0} \simeq \beta+1$. FBG2 is the complementary filter

$$
\begin{aligned}
H_{2}(w) & =0, w>w_{c} \\
& =g \sqrt{-\left(w-w_{c}\right)} e^{j w \tau}, w_{c}-n_{0} w_{m}<w<w_{c} \\
& =e^{j w \tau}, w<w_{c}-n_{0} w_{m} .
\end{aligned}
$$

The power responses $\left|H_{1}(w)\right|^{2}$ and $\left|H_{2}(w)\right|^{2}$ of the two FBGs are linear in the frequency range of interest. Thus, for FBG1, $h_{n}=\left|H\left(w_{c}+n w_{m}\right)\right|=\sqrt{n / n_{0}}$ for $n \geq 0$ and 0 for $n<0$, so that $j_{n}=\sqrt{n / n_{0}} J_{n}(\beta)$. However, as a result of imperfections in the filters or by intention, $j_{0}$ will in practice be a small nonzero value $j_{0}=\sqrt{y / n_{0}} J_{0}(\beta)$ with $0<y<1$.

In this case, $a_{1}^{2}(t)$ contains dc and harmonics of all orders

$$
a_{1}^{2}(t)=\sum_{i=0}^{\infty} j_{i}^{2}+\sum_{k=1}^{\infty} \sum_{i=0}^{\infty} 2 j_{i} j_{i+k} \cos \left[k\left(w_{m}(t-\tau)\right)\right] .
$$

Similarly, for FBG2, $h_{n}=\left|H\left(w_{c}+n w_{m}\right)\right|=-\sqrt{-n / n_{0}}$ for $n \leq 0$ and 0 for $n>0$ and

$$
a_{2}^{2}(t)=\sum_{i=-\infty}^{0} j_{i}^{2}+\sum_{k=1}^{\infty} \sum_{i=-\infty}^{0} 2 j_{i} j_{i+k} \cos \left[k w_{m}(t-\tau)\right] .
$$

The class B output obtained from the difference $a_{1}^{2}(t)-a_{2}^{2}(t)$ results in cancelation of the dc and even harmonics, and a doubling of the fundamental and odd harmonics

$$
a_{1}^{2}(t)-a_{2}^{2}(t)=\sum_{k=1, k \text { odd }}^{\infty} \sum_{i=0}^{\infty} 4 j_{i} j_{i+k} \cos \left[k w_{m}(t-\tau)\right]
$$

The ratio $r_{k}$ of the $k$ th harmonic to the carrier wave for both $a_{1}^{2}$ and $a_{2}^{2}$ is

$$
r_{k}=\frac{\sum_{i=0}^{\infty} j_{i} j_{i+k}}{\sum_{i=0}^{\infty} j_{i} j_{i+1}}=\frac{\sum_{i=0}^{\infty} h_{i} h_{i+k} J_{i}(\beta) J_{i+k}(\beta)}{\sum_{i=0}^{\infty} h_{i} h_{i+1} J_{i}(\beta) J_{i+1}(\beta)} .
$$

For large $\beta$, these results are expected to correspond to those obtained from a half-wave rectified sine wave [17]

$u(t)=\frac{1}{\pi}+\frac{1}{2} \sin w t-\sum_{k=2, k \text { even }}^{\infty} \frac{2}{\pi}\left(\frac{1}{(k-1)(k+1)} \cos k w t\right)$

so that $r_{2}=4 / 3 \pi, r_{3}=0, r_{4}=4 / 15 \pi, r_{5}=0$, and in general $r_{k}=(4) /(\pi(k-1)(k+1))$ for $k$ even and zero otherwise.

\section{B. Small Modulation Index Analysis}

In this subsection, we find the signal relative to dc for small FM index $\beta \ll 1$ using approximations from the series expansion

$$
J_{n}(\beta)=\sum_{l=0}^{\infty} \frac{(-1)^{l}}{(n+l) !}\left(\frac{\beta}{2}\right)^{2 l+n} .
$$

Fifth-order and higher terms are omitted.

It may be desirable or unavoidable to have some of the carrier leak through the filter, as discussed later. If the leakage at $w_{c}$ is $h_{0}=\sqrt{y / n_{0}}=\sqrt{x} \neq 0$

$$
\begin{aligned}
a_{1}^{2}(t)= & {\left[x\left(1-\beta^{2} / 2\right)+\left(\beta^{2} / 4+\beta^{4} / 32\right)\right.} \\
& +\cos \left(w_{m}(t-\tau)\right)(x(\beta / 2) \\
& \left.+\sqrt{2} \beta^{3} / 8+\sqrt{6} \beta^{5} / 192\right) \\
& +\cos \left(2 w_{m}(t-\tau)\right)\left(x \sqrt{2}\left(\beta^{2} / 8\right)+\sqrt{3} \beta^{4} / 48\right) \\
& +\cos \left(3 w_{m}(t-\tau)\right)\left(x \sqrt{3}\left(\beta^{3} / 48\right)+\beta^{5} / 192\right) \\
& +\cos \left(4 w_{m}(t-\tau)\right)\left(x \beta^{4} / 192+\beta^{5} / 192\right) \\
& \left.+\cos \left(5 w_{m}(t-\tau)\right)\left(x \sqrt{5} \beta^{5} / 3840\right)\right] / n_{0} .
\end{aligned}
$$

The output of FBG2 is identical, except that the fundamental signal and odd harmonics have the opposite sign.

The class B output is doubled at the odd harmonics and canceled to zero at dc and even harmonics.

\section{EXPERIMENTAL SETUP AND APPROACH}

Fig. 3 shows the experimental setup to test the theoretical analysis. The output of the external-cavity tunable (ECT) laser is phase modulated by an RF signal at $f_{m}=w_{m} / 2 \pi=1 \mathrm{GHz}$. The phase-modulated optical signal is then amplified using an Erbium-doped fiber amplifier (EDFA) before being directed towards a fiber-Bragg grating (FBG). The specially designed FBG works as an optical frequency discriminator (OFD) with a linear transfer function (power reflectivity versus optical frequency) over $10 \mathrm{GHz}$, shown as inset in Fig. 3 . Thus, $n_{0}=10$, and clipping of significant sidebands will occur for $\beta>9$.

The FBGs were fabricated by Redfern Optical Components, targeting constant group delay and a linear power reflectivity $R$ over a $10-\mathrm{GHz}$ frequency ramp. Maximum $R$ of each $13-\mathrm{cm}-$ 


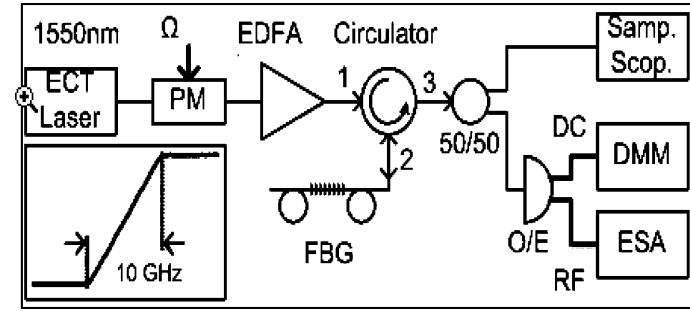

Fig. 3. Experimental setup. The inset shows the FBG power response.

long grating is $94 \%$. Group delay variations are typically $\pm 10 \mathrm{ps}$ over the linear portions of the response curve, with larger variations at the corners and over the portions with flat frequency response.

The RF spectra of the reflected light from OFD are measured using a photodetector $(\mathrm{O} / \mathrm{E})$ and an electrical spectrum analyzer (ESA), while the waveforms are measured by an optical sampling scope. A practical Class-B link, as reported in [16], consists of two complementary OFD and balanced detection. The link in Fig. 3, with FBG biased at zero, measures effectively the spectral and temporal characteristics of one path of the Class-B link, which is sufficient to validate this analysis.

\section{NUMERICAL AND EXPERIMENTAL RESULTS}

Numerical results are shown in both time and frequency domains, followed by experimental results.

Figs. 4-6 show $a_{1}^{2}(t), a_{2}^{2}(t)$ and $a_{1}^{2}(t)-a_{2}^{2}(t)$ for different values of $\beta$ with $x=0$. We observe that for $\beta=0.5$, each FBG output contains a significant dc component (which cancels in class B), and low harmonic distortion. For $\beta=2.0$ the dc component from each FBG is smaller relative to the signal component, but the odd harmonic distortion is increased. For $\beta=8.0$, the dc component of each FBG output approaches its smallest possible value (half of the peak to peak signal component), but the harmonic distortion in each FBG output is quite apparent. For the latter case, the FBG outputs begin to approximate the expected ideal class B operation. For $\beta>20$ (not shown), the complementary signals appear as half-wave rectified cosine waves, and the output approximates a cosine wave. These results can also be observed using a frequency domain view of the FBG outputs. For the class B output of the balanced detector, the signal and odd harmonics are doubled, and the dc and even harmonics are canceled out.

Values of the dc component, desired signal component (fundamental) and harmonics of $a_{1}^{2}(t)$ are plotted versus $\beta$ in Fig. 7 using (9) with carrier leakage $x=0$ and for $\beta$ up to 0.5 , showing that the signal curve 1 increases with $\beta^{3}$. Fig. 8 shows the corresponding values with $x=0.1$. In this case, the signal curve 1 increases linearly with $\beta$ (from the signal term in (15), the linear term dominates when $\beta^{2}>4 x / \sqrt{2}$ ). Operating with some residual carrier leads to a linear input-output relationship with significant dc, typical of class A links.

Fig. 9 shows values of the dc component, desired signal component (fundamental) and harmonics of $a_{1}^{2}(t)$ versus $\beta$ over a larger range of $\beta$ up to 2.5 and harmonics up to the 5th, with

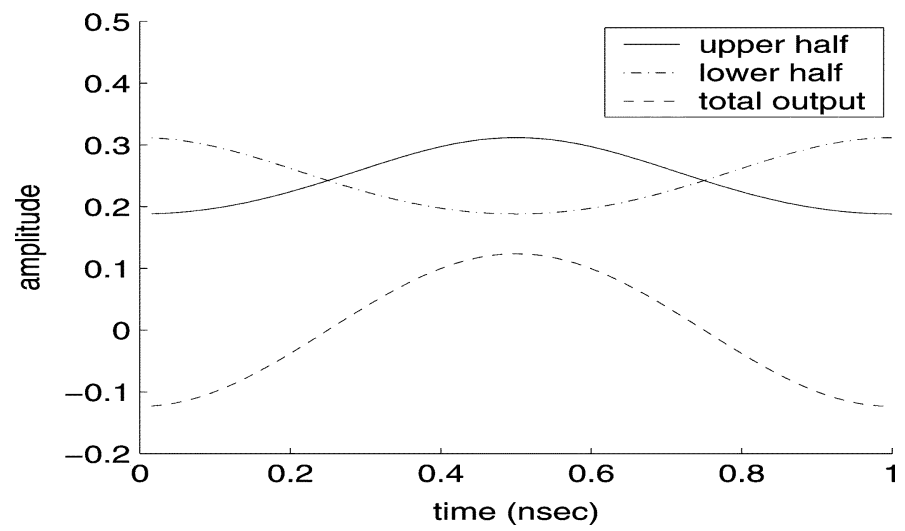

Fig. 4. Class B waveforms, $\beta=0.5$.

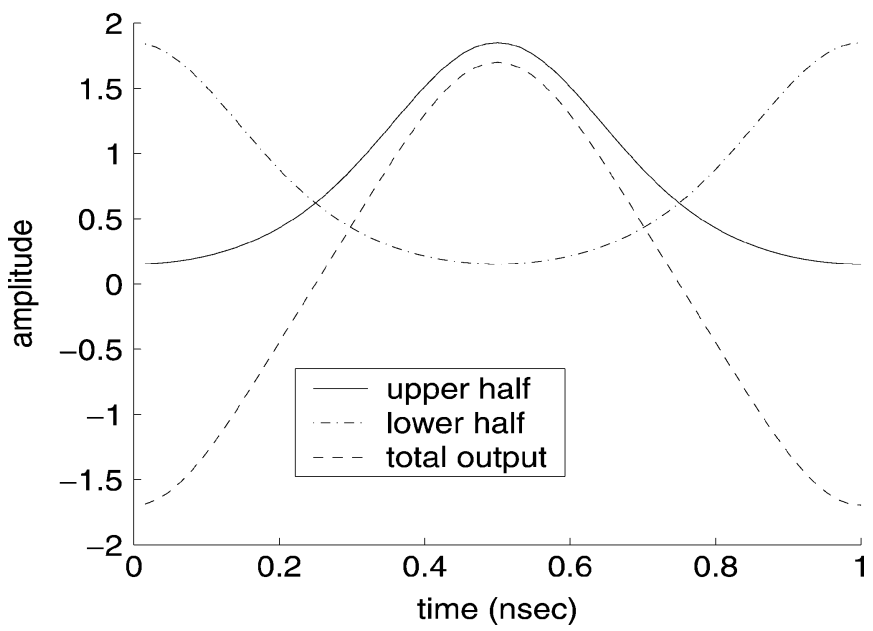

Fig. 5. Class B waveforms, $\beta=2$.

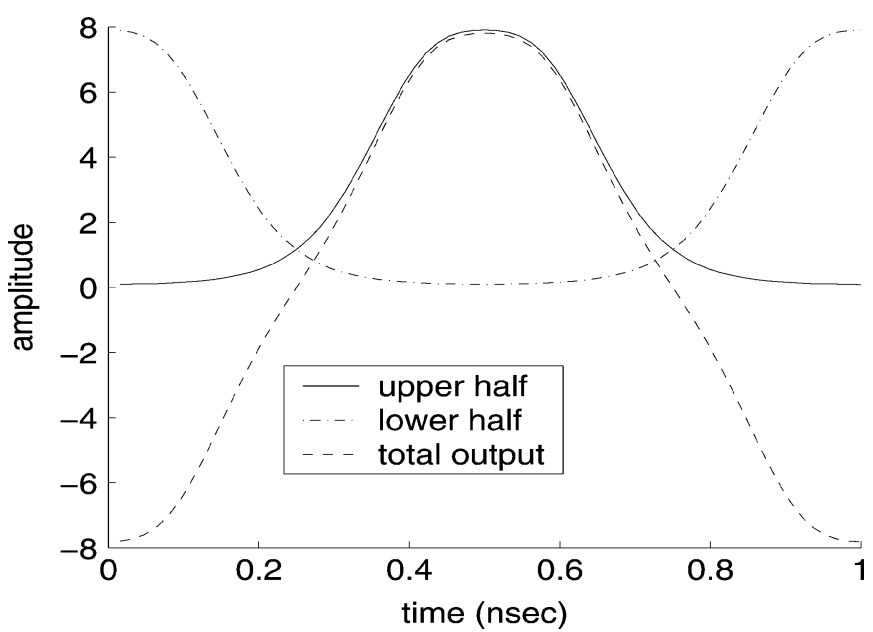

Fig. 6. Class B waveforms, $\beta=8$.

$x=0$. For large $\beta$, the absolute value of power in the odd harmonics increases linearly with $\beta$.

Fig. 10 shows the ratio of the $k$ th harmonic to dc, and Figs. 11 and 12 show the ratio of the $k$ th harmonic to carrier for $k=$ $2,3,4$, and 5 with $x=0$. Fig. 12 is a magnified view of Fig. 11 . The ratios increase with $\beta$ for small $\beta$ to reach a peak value for $\beta$ in the range 3 to 4 . For large $\beta>10$, the ratios approach a 


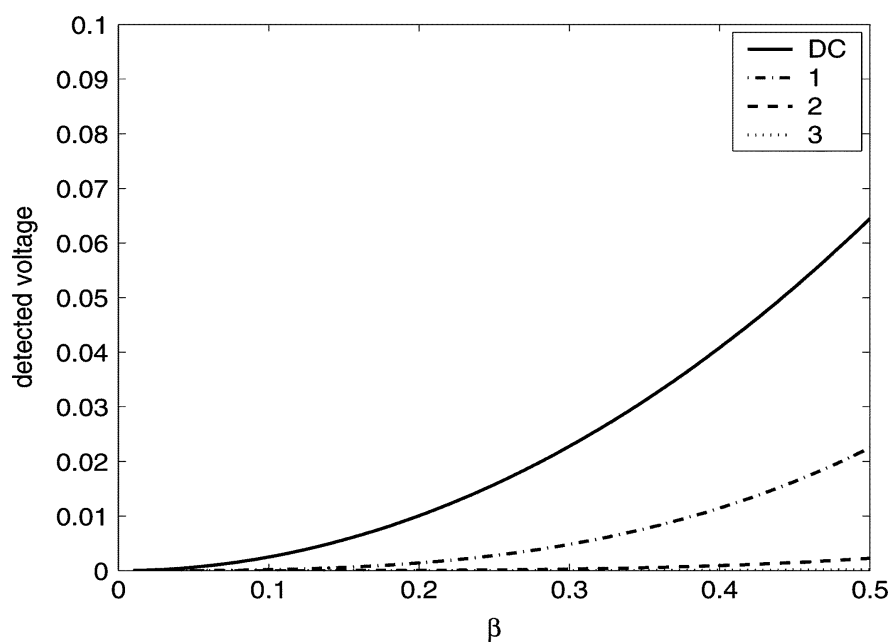

Fig. 7. Detected voltage harmonics versus $\beta$ at one FBG output.

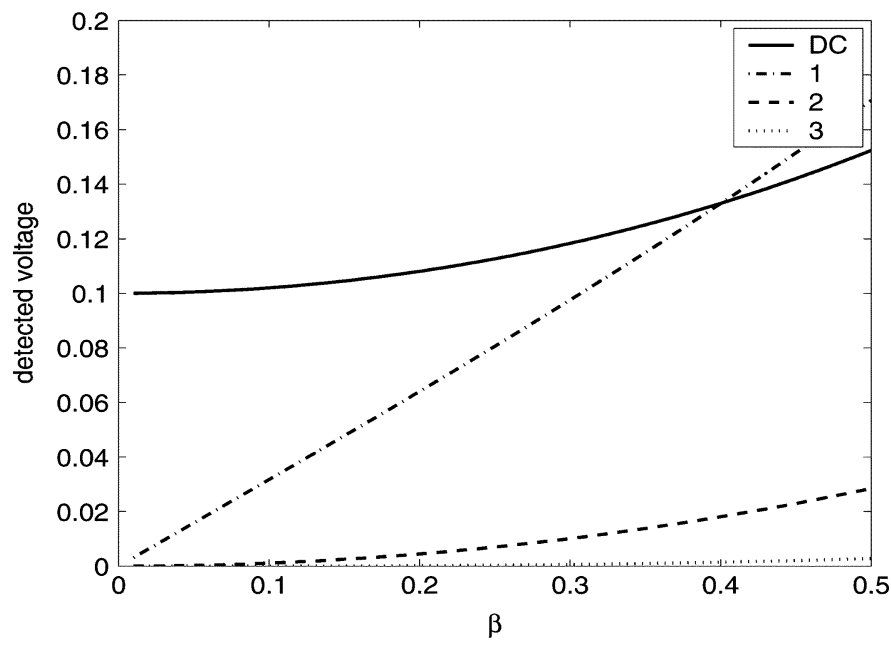

Fig. 8. Detected voltage harmonics versus $\beta$ at one FBG output with carrier leakage.

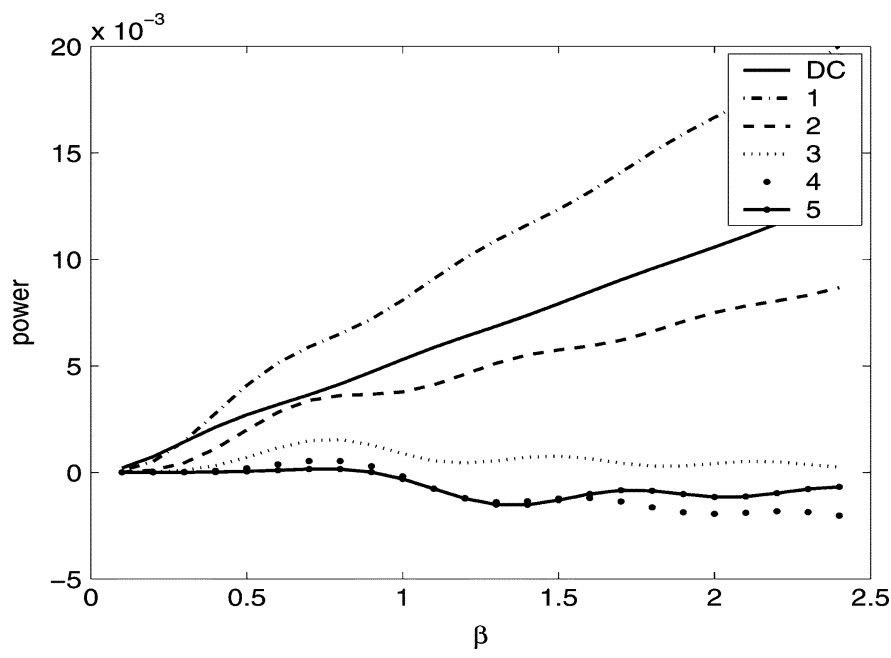

Fig. 9. Optical power (detected voltage) harmonics versus $\beta$ at one FBG output.

constant value near the ratios expected for a half-wave rectified cosine wave.

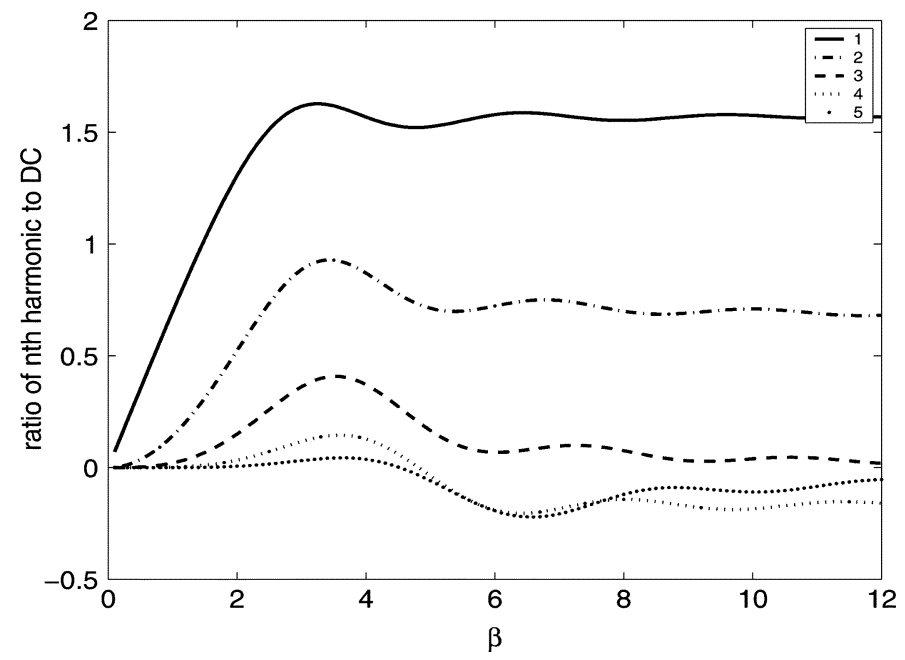

Fig. 10. Ratio of harmonics to de versus $\beta$ at one FBG output.

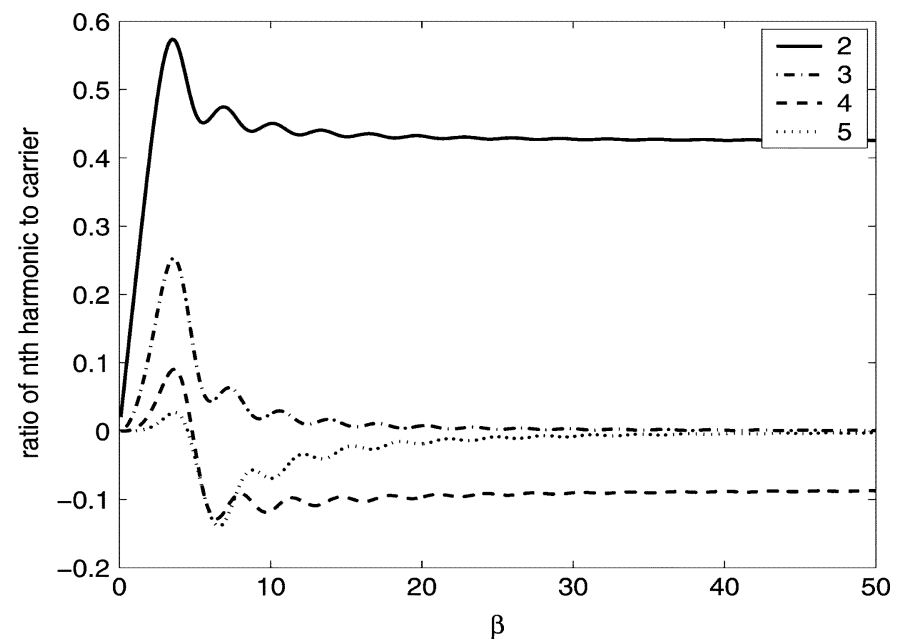

Fig. 11. Ratio of harmonics to signal voltage versus $\beta$ at one FBG output.

In particular, Fig. 10 shows the signal-to-de ratio is as high as it can be for a rectified cosine wave signal. Figs. 11 and 12 show that the third-order harmonic distortion at one FBG output is low for small $\beta$, maximum for intermediate $\beta$, and reduces towards zero as $\beta$ is further increased. Thus, for large $\beta$ the FBG filter shape in (7) simultaneously achieves both the lowest possible dc (i.e., the dc component of a rectified cosine wave) and low third-order harmonic distortion at each FBG output (which leads to maximum SDFR in class B operation [15]). However, this ideal class B result is obtained only for $\beta$ greater than about 20 .

To confirm these theoretical results, Figs. 13-16 show the power of one FBG output from (5) for specified values of $\beta$ and $x$, along with the corresponding measured results in both time and frequency domains. Fig. 13 shows one cycle of the detected voltage for $\beta=0.5, x=0.05$. Aside from a slight phase shift, agreement between theory and experiment is reasonable. Fig. 14 shows the power spectrum of the detected voltage at one FBG output, obtained by taking the fast Fourier transform (FFT) of the five cycles of the time domain waveform that were recorded. The agreement between theory and FFT is excellent; however, the actual frequency domain measurements agree less well but 


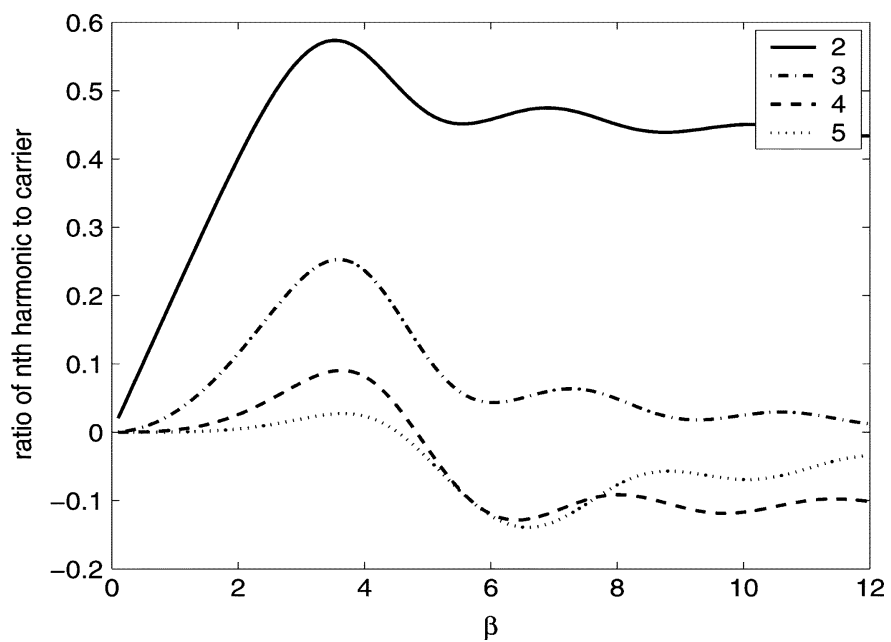

Fig. 12. Ratio of harmonics to signal voltage versus $\beta$ at one FBG output, magnified view of previous figure.

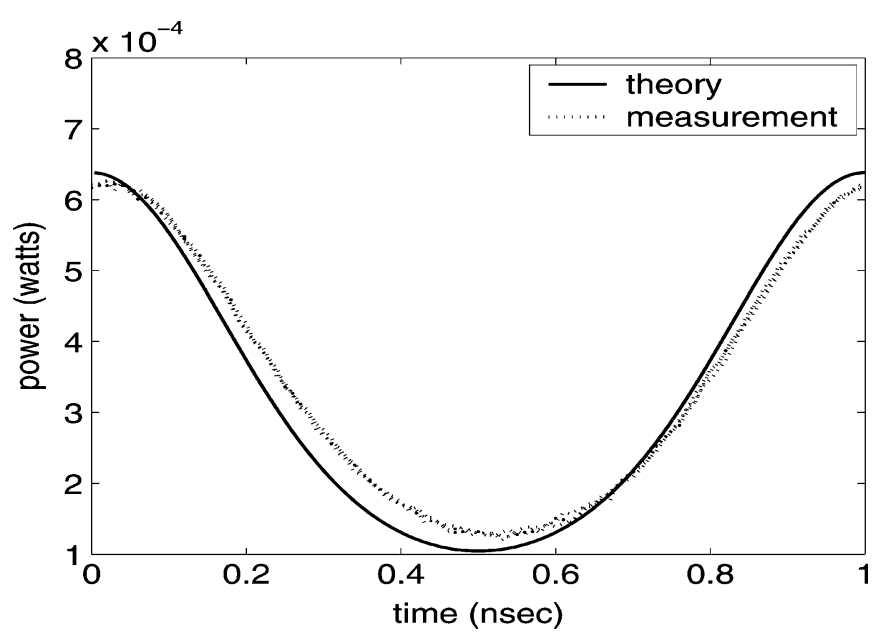

Fig. 13. Detected voltage at one FBG output, $\beta=0.5, x=0.05$.

still reasonably. Fig. 15 shows one cycle of the detected voltage for $\beta=2.0, x=0.05$, and Fig. 16 shows the frequency domain results. Agreement between theory and experiment is similar to that for Figs. 13 and 14. Measurements for $\beta=8$ could not be obtained due to limitations on the RF power, and thus FM clipping was not a factor in the experiments.

The full class B system with two FBGs was not implemented. The single FBG results reported here are of value regardless, since they confirm the analysis showing that ideal class B FM can be achieved with a large modulation index.

\section{DISCUSSION}

Equation (15) shows that for small $\beta$, class B operation results in third-order distortion that is fundamental to the detection process and must be considered in link performance. The ratio of third-order (third harmonic) distortion to signal $(3 H D / S)$, given by (15) and assuming sufficient carrier leakage that the $x$ terms dominate, is $\beta^{2} / 13.7=m^{2} / 3.43$, where the modulation index (signal $/ \mathrm{dc}) m=\beta / 2$. For a quadrature-biased MZ modulator, $3 H D / S=m^{2} / 24$. Therefore, third-order distortion for small $\beta$ is substantially worse than an MZ intensity modulator.

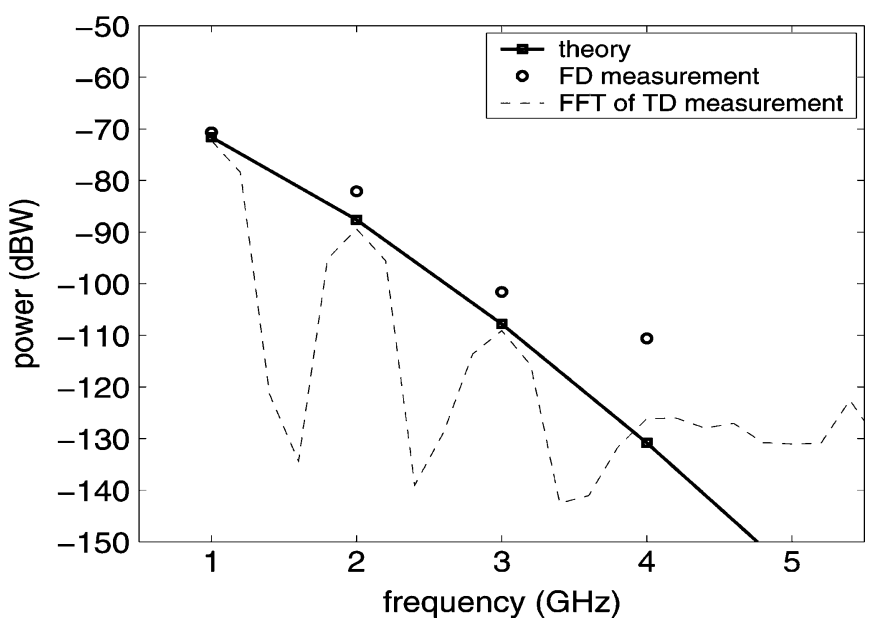

Fig. 14. Power spectrum of detected voltage at one FBG output, $\beta=0.5, x=$ 0.05

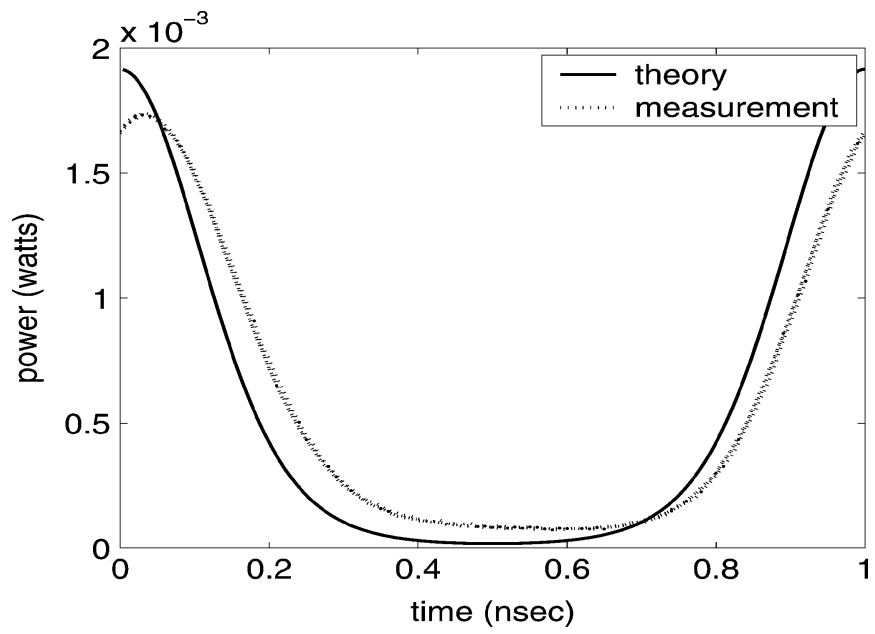

Fig. 15. Detected voltage at one FBG output, $\beta=2.0, x=0.05$.

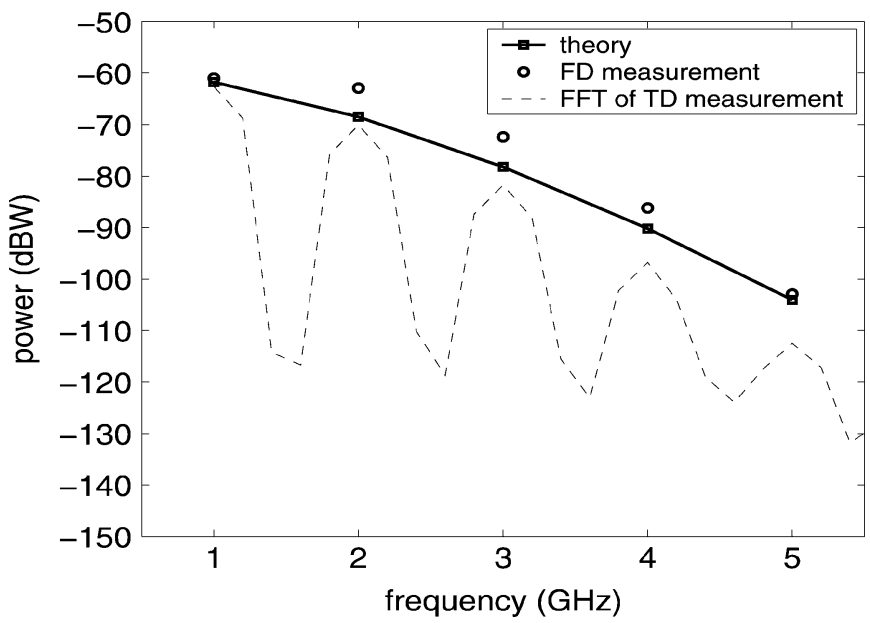

Fig. 16. Power spectrum of detected voltage at one FBG output, $\beta=2.0, x=$ 0.05 .

In contrast, for large $\beta$, class $\mathrm{B}$ operation results in the ratio of third-order distortion to signal reducing towards zero as $\beta$ is increased, and the signal/dc ratio is the $\pi / 2$ value for a half-wave 


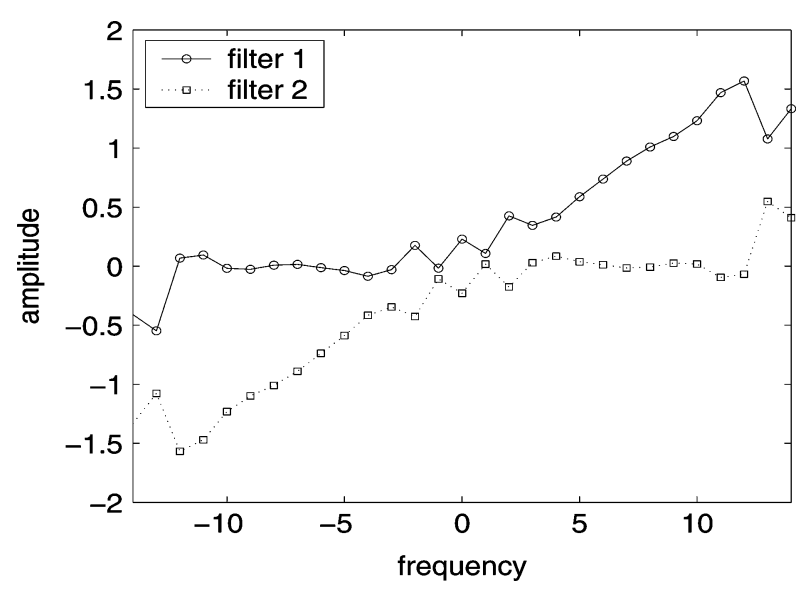

Fig. 17. Optimum class B filter amplitude response for $\beta=8$. The frequency in $\mathrm{GHz}$ is relative to the optical carrier.

rectified cosine wave. Shot noise and RIN are functions of dc and thus are substantially lower for ideal class B operation with large $\beta$ than for conventional links [15].

The essential result is that class B FM does achieve low dc and low third-order harmonic distortion, but only for large $\beta$. This may appear counter-intuitive, since for all values of $\beta$ including small $\beta$, the instantaneous frequency varies sinusoidally above and below $w_{c}$ and thus the FBG output might be expected to be a rectified cosine wave with low dc and low third-order harmonic distortion. However, the results show that for small $\beta$ this is not the case. The reason this intuition fails is that combining a time-domain view of the FM signal (instantaneous frequency, not averaged over time) with a frequency domain view of the FBG filter response is inconsistent with the frequency domain analysis in (2).

In the Appendix, we find an "optimum" filter shape that does achieve both low dc and low third-order distortion simultaneously for any $\beta$ including small $\beta$, but this filter shape depends on $\beta$ and thus is not practical to implement over a range of $\beta$.

\section{CONCLUSION}

A class-B MPL using optical FM and fiber-Bragg gratings is analyzed and results verified with measurements. Input voltage is converted linearly to FM, and this FM is converted by each FBG to intensity modulation (IM). The balanced detector subtracts currents generated in each detector, yielding an effective transfer function with zero dc. The signal increases as $\beta^{3}$ for small $\beta$, becoming linear in $\beta$ with carrier leakage. This carrier leakage makes the class $\mathrm{B}$ link behave more like a suppressed carrier class A link, and may generally provide adequate reduction in dc-related noise. We have quantified the level of third-order distortion that results from the sideband filtering operation, and shown that for small $\beta$, this is worse than for a MZ intensity modulator. However, for large $\beta$, class B operation operates as expected, simultaneously achieving low dc and low third-order distortion, and thus substantially reduces IM3, shot noise, and RIN, as reported in [15]. Ideal class B operation can be realized for large index FM modulation only.

\section{APPENDIX}

In this Appendix, we consider a more optimum filter shape $h_{n}$ which achieves both low dc and low third-order harmonic distortion for both large and small $\beta$. The desired filter output will have an envelope appearing as a rectified cos wave $s_{\text {rectcosFM }}(t)=s_{\text {rectcos }}(t) \cdot s_{\mathrm{FM}}(t)$. An FM signal with an exact rectified cosine envelope has a spectrum $S_{\text {rectcosfM }}(f)=S_{\text {rectcos }}(f) \otimes S_{\mathrm{FM}}(f)$. The ratio $H_{\mathrm{opt}}(f)=S_{\text {rectcosFM }}(f) / S_{\mathrm{FM}}(f)$ is the transfer function of a filter which yields $s_{\text {rectcosFM }}(t)$ in response to input $s_{\mathrm{FM}}(t)$. Note that $H_{\text {opt }}$ has a nonzero response on both sides of the carrier, and is optimum for only one particular value of $\beta$. For large $\beta=8$ (Fig. 17), the optimum filter begins to approximate the FBG transfer function (7) shown in Fig. 2(a).

\section{REFERENCES}

[1] C. H. Cox III, Analog Optical Links: Theory and Practice. Cambridge, U.K.: Cambridge Univ. Press, 2004.

[2] T. E. Darcie and M. R. Phillips, "Lightwave Video Transmission," in Optical Fiber Telecommunications IIIA. New York: Academic, 1997, chapter in.

[3] L. T. Nichols, K. J. Williams, and R. D. Esman, "Optimizing the ultrawide-band photonic link," IEEE Trans. Microw. Theory Tech., vol. 45, no. 8, pp. 1384-1389, Aug. 1997.

[4] V. J. Urick, M. S. Rogge, P. F. Knapp, L. Swingen, and F. Bucholtz, "Wide-band predistortion linearization for externally modulated longhaul analog fiber-optic links," IEEE Trans. Microw. Theory Tech., vol. 54, no. 4, pp. 1458-1463, Jun. 2006.

[5] B. Liu, J. Shim;, Y.-J. Chiu, A. Keating, J. Piprek, and J. E. Bowers, "Analog characterization of low-voltage MQW traveling-wave electroabsorption modulators," J. Lightw. Technol., vol. 21, no. 12, pp. 3011-3019, Dec. 2003.

[6] A. A. M. Saleh, "Fundamental limit on number of channels in subcarrier-multiplexed lightwave CATV system," Electron. Lett., vol. 25, no. 12, pp. 776-777, Jun. 8, 1989.

[7] J. Zhang, A. N. Hone, and T. E. Darcie, "Limitation due to signal-clipping in linearized microwave-photonic links," IEEE Photon. Technol. Lett., vol. 19, no. 14, pp. 1033-1035, Jul. 15, 2007.

[8] M. J. LaGasse and S. Thaniyavaru, "Bias-free high-dynamic-range phase-modulated fiber-optic link," IEEE Photon. Technol. Lett., vol. 9, no. 5, pp. 681-683, May 1997.

[9] J. Zhang and T. E. Darcie, "Low-biased microwave-photonic link using optical frequency or phase modulation and fiber-Bragg-grating discriminator," in Proc. Optical Fiber Commun. Conf. National Fiber Optic Eng. Conf., Mar. 5-10, 2006, p. 3.

[10] J. E. Bowers, A. Ramaswamy, L. A. Johansson, J. Klamkin, M. Sysak, D. Zibar, L. Coldren, M. Rodwell, L. Lembo, R. Yoshimitsu, D. Scott, R. Davis, and P. Ly, "Linear coherent receiver based on a broadband and sampling optical phase-locked loop," in IEEE Int. Topical Meeting Microw. Photon., Oct. 3-5, 2007, pp. 225-228.

[11] H. V. Roussell, M. D. Regan, J. L. Prince, C. H. Cox, J. X. Chen, W. K. Burns, G. E. Betts, E. I. Ackerman, and J. C. Campbell, "Gain, noise figure and bandwidth-limited dynamic range of a low-biased external modulation link," in Proc. IEEE Int. Topical Meeting Microw. Photon., Oct. 3-5, 2007, pp. 84-87.

[12] R. D. Esman and K. J. Williams, "Wideband efficiency improvement of fiber optic systems by carrier subtraction," IEEE Photon. Technol. Lett., vol. 7, no. 2, pp. 218-220, Feb. 1995.

[13] W. K. Burns, G. K. Gopalakrishnan, and R. P. Moeller, "Multi-octave operation of low-biased modulators by balanced detection," IEEE Photon. Technol. Lett., vol. 8, no. 1, pp. 130-132, Jan. 1996.

[14] J. D. Bull;, T. E. Darcie;, J. Zhang;, H. Kato;, and N. A. F. Jaeger, "Broadband class-AB microwave-photonic link using polarization modulation," IEEE Photon. Technol. Lett., vol. 18, no. 9, pp. 1073-1075, May 1, 2006.

[15] T. E. Darcie; and P. F. Driessen, "Class-AB techniques for high-dynamic-range microwave-photonic links," IEEE Photon. Technol. Lett., vol. 18, no. 8, pp. 929-931, Apr. 2006. 
[16] T. E. Darcie, J. Zhang;, P. F. Driessen, and J.-J. Eun, "Class-B microwave-photonic link using optical frequency modulation and linear frequency discriminators," J. Lightw. Technol., vol. 25, no. 1, pp. 157-164, Jan. 2007.

[17] M. Abramowitz and I. A. Stegun, Handbook of Mathematical Functions. New York: Dover, p. 363, 9.1.77, (online).

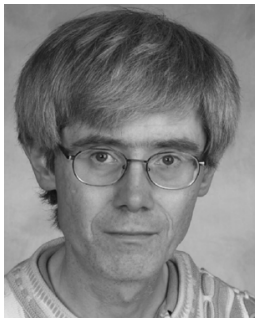

Peter F. Driessen (SM'93) received the B.Sc. degree in physics and the Ph.D. degree in electrical engineering from the University of British Columbia, Vancouver, BC, Canada, in 1976 and 1981, respectively.

He was with various companies in Vancouver designing modems for five years before joining the University of Victoria, Victoria, BC, where he is currently Professor at the Department of Electrical and Computer Engineering and cross-appointed to the Department of Computer Science and the School of Music. He spent two sabbaticals and several summers at AT\&T Bell Laboratories, Holmdel, NJ, working on various aspects of wireless communications systems. He has more than 100 technical publications and 11 patents. His research interests include microwave-photonics, audio and video signal processing, computer music, sound recording, wireless communications, and radio propagation.

Dr. Driessen was an Editor for the IEEE TRANSACTIONS ON WIRELESS COMMUNICATIONS from 1999 to 2004.

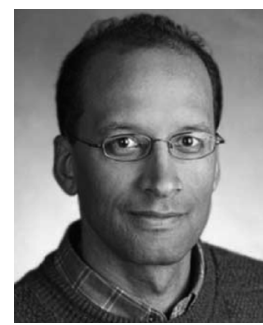

Thomas E. Darcie (F'99) received the B.Sc. degree in physics from the University of Waterloo, Waterloo, ON, Canada, in 1978 and the M.Sc. and Ph.D. degrees in aerospace from the University of Toronto, Toronto, ON, in 1982.

He joined the technical staff of AT\&T Bell Laboratories, Holmdel, NJ, to study a wide variety of topics related to lightwave telecommunications, including fiber fabrication processes, semiconductor lasers, optical amplifiers, and numerous modulation and multiplexing techniques. He has been a lead figure in the development of lightwave systems for analog applications in cable television and wireless systems. As the Head of access communications research at AT\&T Bell Laboratories from 1989 to 1995, he was responsible for technology innovation in wireless, lightwave, and hybrid fiber-coax systems. As Vice President of AT\&T Laboratories and Director of the Communications Infrastructure Research Laboratory from 1995 to 2002, he led a research laboratory that provided technology support for AT\&T's diverse requirements in optical networking, broadband access, fixed wireless access, wireless local-area networks, and cellular systems. He is currently a Professor at the University of Victoria, Victoria, BC, Canada, and holds a Tier 1 Canada Research Chair. He has more than 100 technical publications and 30 patents spanning the aforementioned broad set of technologies. His research program focuses on systems for communications, imaging, and sensor applications.

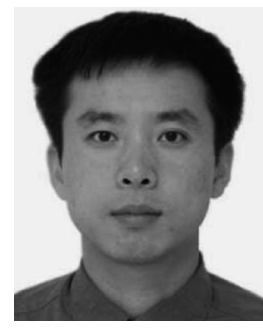

Jinye Zhang (M'05) received the B.Sc. and M.Sc. degrees in physico-electronics from Tianjin University, Tianjin, China, in 1994 and 2000, respectively, and the Ph.D. degree in electrical engineering from the Northwestern University, Evanston, IL, in 2005.

Since 2005, he has been a Postdoctoral Fellow at the Optical Systems and Technology Laboratory, University of Victoria, Victoria, BC, Canada. His research interests include nonlinearities in optical fibers and fiber-optic systems for communications and sensing.

Dr. Zhang is a member of the Optical Society of America. 